\title{
A Dalbavancin Lock Solution Can Reduce Enterococcal Biofilms After Freezing
}

\author{
Marta Díaz-Navarro • Rama Hafian · Irene Manzano • María J. Pérez-Granda • \\ Emilia Cercenado • Cristina Pascual · Carmen Rodríguez • \\ Patricia Muñoz · María Guembe (D)
}

Received: October 21, 2021 / Accepted: December 1, 2021 / Published online: January 16, 2022

(C) The Author(s) 2021

\section{ABSTRACT}

Introduction: We previously demonstrated the efficacy of a frozen dalbavancin-heparin (DH) lock solution against biofilms of staphylococci. However, as enterococci also commonly cause

M. Díaz-Navarro · M. J. Pérez-Granda · E. Cercenado · P. Muñoz · M. Guembe ( $\square)$

Department of Clinical Microbiology and Infectious Diseases, Hospital General Universitario Gregorio Marañón, C/Dr. Esquerdo, 46, 28007 Madrid, Spain e-mail: mariaguembe@hotmail.com; maria.guembe@iisgm.com

M. Díaz-Navarro · M. J. Pérez-Granda · P. Muñoz · M. Guembe

Instituto de Investigación Sanitaria Gregorio

Marañón, Madrid, Spain

R. Hafian · I. Manzano

Biology Department, School of Biology, Universidad Complutense de Madrid, Madrid, Spain

M. J. Pérez-Granda · P. Muñoz

CIBER Enfermedades Respiratorias-CIBERES (CB06) 06/0058), Madrid, Spain

E. Cercenado · P. Muñoz

Medicine Department, School of Medicine,

Universidad Complutense de Madrid, Madrid, Spain

C. Pascual

Hematology Department, Hospital General

Universitario Gregorio Marañón, Madrid, Spain

C. Rodríguez

Pharmacy Department, Hospital General

Universitario Gregorio Marañón, Madrid, Spain catheter-related bloodstream infections (CRBSI), we assessed the bioactivity of frozen dalbavancin (D) and DH against enterococci.

Methods: Over 6 months, we compared the bioactivity of a solution of $\mathrm{DH}(1 \mathrm{mg} / \mathrm{ml})$ with that of $\mathrm{D}$ in terms of cfu counts and metabolic activity against biofilms of Enterococcus faecalis and Enterococcus faecium (four strains each). For each solution, we individually compared results obtained at each time point (months 3 and 6) with baseline (month 0 ). We also compared the median $\mathrm{DH}$ value of each variable at baseline and at months 3 and 6 of freezing with the values obtained for $\mathrm{D}$ alone. We used both statistical and clinical criteria when results were within $25 \%$ of the reference value.

Results: At the end of the experiment (month 6), neither a statistically nor a clinically significant reduction in the bioactivity of $\mathrm{D}$ solution was observed in terms of cfu count and metabolic activity against enterococcal biofilms. Regarding the DH solution, we found both statistical and clinical significance in the median percentage reduction in metabolic activity between months 0 and 6 in E. faecalis strains (51.8\% vs. $77.8 \%, P=0.007)$. Moreover, after freezing, the DH solution lost significant bioactivity compared with the D solution, especially in E. faecalis.

Conclusion: A dalbavancin lock solution can be frozen for up to 6 months with no negative effect on its bioactivity against enterococcal biofilms. However, when combined with heparin, its 
efficacy was reduced. Therefore, we recommend that if lock therapy with frozen dalbavancin is used in the management of enterococcal C-RBSI, heparin should be added simultaneously at the time of catheter lock.

Keywords: Biofilms; Dalbavancin; Enterococci; Freezing; Heparin; Lock therapy

\section{Key Summary Points}

\section{Why carry out this study?}

Data regarding the activity of dalbavancin as catheter lock solution against enterococci strains is scarce.

We assessed whether the activity of a frozen dalbavancin catheter lock solution could be affected by the addition of heparin against enterococcal biofilms.

\section{What was learned from the study?}

The activity of a dalbavancin catheter lock solution was not affected by a 6-month freezing.

However, the addition of heparin to a dalbavancin catheter lock solution negatively affects its activity.

\section{INTRODUCTION}

Catheter-related bloodstream infection (C-RBSI) is a major nosocomial infection that leads to high morbidity and mortality rates $[1,2]$. While catheter withdrawal followed by systemic antimicrobial therapy is best practice for treatment, conservative management with catheter lock therapy has proven successful in several clinical studies [3-13]. However, as current guidelines for the management of C-RBSI are somewhat outdated, they do not provide data on new antibiotics that can be used in catheter lock solutions $[14,15]$.

Dalbavancin (D) is a lipoglycopeptide that is highly active against Gram-positive bacteria and has a proven marked anti-biofilm effect, in particular, better than oritavancin and comparable to telavancin [16]. In previous studies, we tested the efficacy of D against Staphylococcus spp. and found it to be noninferior to vancomycin in terms of reduction in the values of specific parameters associated with biofilm [17]. Moreover, in order to optimize health costs, we demonstrated that a D lock solution could be frozen for up to 6 months without reducing its bioactivity [18].

Our objectives were, first, to validate our findings against enterococcal biofilms, as enterococci also frequently cause C-RBSI. Second, we assessed whether heparin could affect the activity of D.

\section{METHODS}

The study was performed in the laboratory of the Department of Clinical Microbiology and Infectious Diseases at Gregorio Marañón Hospital, Madrid, Spain.

We ran an in vitro static microplate model similar to our previous work [18] using three clinical strains of Enterococcus faecalis and three clinical strains of Enterococcus faecium causing C-RBSI and ATCC strains of Enterococcus faecalis ATCC33186 and Enterococcus faecium ATCC19434, all of which are high biofilm producers. The selection of the six clinical strains was based on their high ability to form biofilms and that they were isolated from patients with C-RBSI, belonging to a large sample collection used in our previous studies [19].

The minimal inhibitory concentration (MIC), assessed using the E-test, of vancomycin-dalbavancin for E. faecalis and E. faecium ATCC strains were, respectively, $2-0.064 \mathrm{mg} / \mathrm{L}$ and $1-0.032 \mathrm{mg} / \mathrm{L}$.

Biofilms were tested at month 0 (baseline before freezing) and at months 3 and 6 of freezing.

As thawed solutions had to be tested against a static biofilm in microplates, frozen $\mathrm{D}$ and dalbavancin-heparin (DH) solutions were thawed at room temperature (30 min). For each solution, we individually compared the results obtained at each time point with baseline. We 
also compared the results for $\mathrm{DH}$ with those obtained for $\mathrm{D}$ at each time point.

The anti-biofilm activity of $\mathrm{D}$ and $\mathrm{DH}$ solutions was performed by adding $100 \mu \mathrm{l}$ of each of them to dry plates followed by incubation at $37^{\circ} \mathrm{C}$ for $24 \mathrm{~h}$. The plates were washed three times with phosphate-buffered saline (PBS) and dried at room temperature [20].

Data were expressed as percentage reduction in $\log$ colony-forming units $(\mathrm{cfu}) / \mathrm{ml}$ and metabolic activity.

\section{Biofilm Formation}

Biofilm was formed as described by Peeters et al. and by our group [18, 21]. Each strain was mixed in $20 \mathrm{ml}$ of tryptic soy broth supplemented with glucose $1 \%$ and incubated at $37^{\circ} \mathrm{C}$ in an orbital shaker for $24 \mathrm{~h}$. The inoculum was then washed with PBS, and the pellets were adjusted to $0.5 \mathrm{McF}$ arland turbidity. Then, $100 \mu \mathrm{l}$ of the suspension was inoculated in a 96-well plate for analysis and plates were incubated at $37^{\circ} \mathrm{C}$ during $24 \mathrm{~h}$, followed by three washes with PBS. We performed experiments in triplicates, including positive (bacterial suspension without treatment) and negative controls (broth medium without bacterial suspension).

\section{Dalbavancin Lock Solution and Treatment Procedure}

We prepared a catheter lock solution of $\mathrm{D}$ of $1 \mathrm{mg} / \mathrm{ml}$ (as guidelines recommend that lock solutions must be 100 or 1000 times the MIC) [14]. We used Xydalba ${ }^{\circledR} 500 \mathrm{mg}$ powder for infusion (Almac Pharma Services Ltd, Seagoe Industrial Estate, Craigavon, County Armagh, BT63 5UA, UK; Allergan Pharmaceuticals International Ltd., Clonshaugh Business \& Technology Park, Dublin 17, D17 E400, Ireland. Almac Pharma Services, Ireland. Limited Finnabair Industrial Estate, Dundalk, Co. Louth, A91 P9KD, Ireland). Xydalba ${ }^{\circledR}$ was reconstituted following the manufacturer's instructions.

From the reconstituted $\mathrm{D}(20 \mathrm{mg} / \mathrm{ml})$, we prepared a DH solution by transferring $1 \mathrm{ml}$ to $18.6 \mathrm{ml}$ of glucose saline $5 \%+0.4 \mathrm{ml}$ heparin sodium (60 IU), heparin sodium $5000 \mathrm{IU} / 5 \mathrm{ml}$
(Hospira Prod. Farm. y Hosp, S.L., Spain). We also prepared a D solution by transferring $1 \mathrm{ml}$ to $19 \mathrm{ml}$ of glucose saline $5 \%$. We stored $3-\mathrm{ml}$ aliquots of each solution at $-70^{\circ} \mathrm{C}$ for 6 months.

\section{Quantification of Bacterial Load by cfu/ml}

Wells were vigorously scraped in $100 \mu \mathrm{l}$ of PBS, serial dilutions were performed, and $100 \mu \mathrm{l}$ of each dilution was cultured into blood agar plates and incubated at $37^{\circ} \mathrm{C}$ for $24 \mathrm{~h}$.

Colonies were counted, and we calculated the percentage reduction in cfu/well using Eq. 1. Results were expressed as median (IQR) $\log \mathrm{cfu} / \mathrm{ml}$.

$\%$ of viability reduction

$$
=\left[1-\left(\frac{\frac{\text { c.f.u. }}{\text { well }} \text { treated strain }}{\frac{\text { c.f.u. }}{\text { well }} \text { positive control }}\right)\right] \times 100 \text {. }
$$

\section{Quantification of Metabolic Activity by XTT Assay}

One hundred microliters of XTT (2,3-bis-(2methoxy-4-nitro-5-sulfophenyl)-2H-tetrazolium-5-carboxanilide)/menadione $(0.5 \mathrm{mg} / \mathrm{ml}$ and $1.72 \mathrm{mg} / \mathrm{ml}$, respectively) was inoculated in each well protected from light and plates were incubated at $37^{\circ} \mathrm{C}$ for $3 \mathrm{~h}$. We measured absorbance in a spectrophotometer (Biochrom EZ Read 400) at $492 \mathrm{~nm}$ and results were expressed as percentage reduction in metabolic activity calculated following Eq. 2 .

$\%$ of metabolic reduction

$$
=\left[1-\left(\frac{\text { Abs }_{492} \text { treated strain }}{\text { Abs }_{492} \text { positive control }}\right)\right] \times 100 .
$$

Absence of reduction in the anticoagulant effect of $\mathrm{DH}$ before and during a 6-month freezing period has already been demonstrated using the anti-Xa assay in a previous study by our group [18]. 
Table 1 Median (IQR) percentage reduction in $\log \mathrm{cfu} / \mathrm{ml}$ and metabolic activity for dalbavancin solution against Enterococcus faecalis and Enterococcus faecium during freezing

\begin{tabular}{lllll}
\hline Month & Median (IQR) \% reduction in $\log \mathbf{c f u} / \mathbf{m l}$ & $\boldsymbol{P}^{*}$ & Median (IQR) \% reduction in XTT & $\boldsymbol{P}^{*}$ \\
\hline \multicolumn{2}{l}{ Enterococcus faecalis } \\
0 & $22.9(19.8-30.8)$ & $\mathrm{NA}^{\mathrm{a}}$ & $81.9(56.6-89.8)$ & $\mathrm{NA}^{\mathrm{b}}$ \\
3 & $22.8(20.1-34.5)$ & 0.31 & $91.9(89.7-99.7)$ & 0.02 \\
6 & $30.9(19.2-37.4)$ & 0.27 & $95.9(90.5-98.7)$ & 0.003 \\
Enterococcus faecium & & & \\
0 & $22.7(14.1-24.9)$ & $\mathrm{NA}^{\mathrm{c}}$ & $54.7(39.1-68.6)$ & $\mathrm{NA}^{\mathrm{d}}$ \\
3 & $13.1(8.1-30.5)$ & $\mathbf{0 . 5 7}$ & $78.5(30.8-84.2)$ & 0.45 \\
6 & $24.9(21.6-34.2)$ & 0.15 & $98.5(59.2-100)$ & 0.02 \\
\hline
\end{tabular}

$S D$ standard deviation, $c f u$ colony-forming units, $N A$ not applicable, $I Q R$ interquartile range

*Values in bold represent clinical significance (median \% reduction of variables at month 3 or 6 were below $25 \%$ lower limit of median \% reduction of variables at month 0 )

${ }^{\mathrm{a}} 25 \%$ lower limit of median \% reduction of $\log \mathrm{cfu} / \mathrm{ml}$ in E. faecalis at month $0,17.2 \%$

${ }^{\mathrm{b}} 25 \%$ lower limit of median \% reduction of XTT in E. faecalis at month 0, 61.4\%

' $25 \%$ lower limit of median \% reduction of $\log \mathrm{cfu} / \mathrm{ml}$ in E. faecium at month $0,17.0 \%$

$\mathrm{d}_{25 \%}$ lower limit of median \% reduction of XTT in E. faecium at month 0, $41.0 \%$

\section{Clinical and Statistical Analysis}

Quantitative variables are expressed as the median and interquartile range (IQR).

For the comparisons between baseline (month 0 ) and freezing periods (months 3 and 6) with respect to both solutions, we defined clinical significance when the percentage reduction values for each variable tested at each time point were below $25 \%$ of those obtained at baseline. For the comparison between $\mathrm{DH}$ and dalbavancin solution, we defined clinical significance when the percentage reduction values for each variable tested with $\mathrm{DH}$ solution were below $25 \%$ of those obtained with dalbavancin solution at each time point.

We arbitrarily chose a $25 \%$ cutoff, as in our previous study [18], since we considered it a reasonable value above which clinical impact would be relevant.

Although our main objective was to assess clinical significance, we also assessed statistical significance. For each solution, we used the Kruskal-Wallis test to compare the baseline (month 0) median (IQR) percentage reduction values in $\log \mathrm{cfu} / \mathrm{ml}$ and metabolic activity with the values obtained at months 3 and 6 . We also used the Kruskal-Wallis test to compare the median (IQR) percentage reduction values in $\log \mathrm{cfu} / \mathrm{ml}$ and metabolic activity of $\mathrm{DH}$ solution with the values obtained with dalbavancin solution at each time point (months 0,3 , and 6).

Statistical significance was set at $P<0.05$ for all the tests. The statistical analysis was performed using IBM SPSS Statistics for Windows, Version 21.0 (IBM Corp, Armonk, New York, USA).

\section{Ethics}

The study was considered to be exempt for ethical approval by our local ethics committee since it was an in vitro retrospective study. 



Fig. 1 Median ( $\mathrm{QQR}$ ) percentage reduction in $\log \mathrm{cfu} / \mathrm{ml}$ and metabolic activity for dalbavancin solution against enterococci according to freezing time. a E. faecalis. b E. faecium. cfu colony-forming units

\section{RESULTS}

\section{Dalbavancin Solution}

At the end of the experiment (month 6), neither a statistically nor a clinically significant reduction in the bioactivity of dalbavancin solution against enterococcal biofilms was observed in terms of $\log \mathrm{cfu} / \mathrm{ml}$ or metabolic activity. Only a clinically significant reduction (below 25\% of baseline value) was observed (less than 17.0\%) in median (IQR) percentage of $\mathrm{cfu} / \mathrm{ml}$ between month 0 and 3 in E. faecium (13.1\% [8.1-30.5\%] vs. $22.7 \%$ [14.1-24.9\%]) (Table 1, Fig. 1a, b). 
Table 2 Median (IQR) percentage reduction in $\log \mathrm{cfu} / \mathrm{ml}$ and metabolic activity for dalbavancin-heparin solution against Enterococcus faecalis and Enterococcus faecium during freezing

\begin{tabular}{|c|c|c|c|c|}
\hline Month & Median (IQR) \% reduction in $\log \mathrm{cfu} / \mathrm{ml}$ & $P^{*}$ & Median (IQR) \% reduction in XTT & $P^{*}$ \\
\hline \multicolumn{5}{|c|}{ Enterococcus faecalis } \\
\hline 0 & $10.7(4.3-19.4)$ & $\mathrm{NA}^{\mathrm{a}}$ & $77.8(60.7-80.3)$ & $\mathrm{NA}^{\mathrm{b}}$ \\
\hline 3 & $13.0(9.2-19.1)$ & 0.51 & $79.2(70.9-83.0)$ & 0.46 \\
\hline 6 & $9.1(7.9-12.5)$ & 0.69 & $51.8(6.60-61.6)$ & 0.007 \\
\hline \multicolumn{5}{|c|}{ Enterococcus faecium } \\
\hline 0 & $13.2(11.9-20.6)$ & $N A^{c}$ & $84.4(71.8-93.5)$ & $\mathrm{NA}^{\mathrm{d}}$ \\
\hline 3 & $13.7(0.0-21.9)$ & 0.69 & $78.9(71.3-86.7)$ & 0.66 \\
\hline 6 & $13.6(13.0-18.9)$ & 0.79 & $70.7(55.3-85.8)$ & 0.09 \\
\hline
\end{tabular}

$S D$ standard deviation, $c f u$ colony-forming units, $N A$ not applicable, $I Q R$ interquartile range

*Values in bold represent clinical significance (median \% reduction of variables at month 3 or 6 were below $25 \%$ lower limit of median \% reduction of variables at month 0 )

${ }^{\mathrm{a}} 25 \%$ lower limit of median \% reduction of $\log \mathrm{cfu} / \mathrm{ml}$ in E. faecalis at month $0,8.03 \%$

${ }^{\mathrm{b}} 25 \%$ lower limit of median \% reduction of XTT in E. faecalis at month 0, 58.4\%

${ }^{c} 25 \%$ lower limit of median \% reduction of $\log \mathrm{cfu} / \mathrm{ml}$ in E. faecium at month $0,9.9 \%$

$\mathrm{d}_{25 \%}$ lower limit of median \% reduction of XTT in E. faecium at month $0,63.3 \%$

\section{DH Solution}

When dalbavancin was combined with heparin during freezing, the only statistically and clinically significant reduction at the end of the experiments with respect to baseline was for median (IQR) percentage of metabolic activity of E. faecalis $(51.8 \%$ [6.6-61.6\%] vs. $77.8 \%$ [60.7-80.3\%], $P=0.007 ; 25 \%$ of baseline, $58.4 \%$ ) (Table 2, Fig. 2a, b).

\section{Comparison Between Dalbavancin and DH}

When we analyzed whether heparin could affect the bioactivity of dalbavancin, we found that the median (IQR) percentage reduction in $\log \mathrm{cfu} / \mathrm{ml}$ and in metabolic activity was significantly reduced in E. faecalis. Statistically and clinically significant results were observed in median (IQR) percentage reduction in log cfu/ $\mathrm{ml}$ at each time point between the two solutions (Table 3, Fig. 3a). Regarding the median
(IQR) percentage reduction in metabolic activity, clinically significant differences were observed between the $\mathrm{DH}$ and dalbavancin solutions only at month $6(74.8 \%$ [61.3-86.0\%] vs. $95.9 \%$ [90.5-98.7\%]; $25 \%$ of dalbavancin value, $71.0 \%$ ) (Table 3, Fig. 3b).

In contrast, in E. faecium biofilms, the only statistically and clinically significant difference between $\mathrm{DH}$ and dalbavancin was observed for the median (IQR) percentage reduction in $\log \mathrm{cfu} / \mathrm{ml}$ at month $6(13.6 \%[13.0-18.9 \%] \mathrm{vs}$. $20.4 \%$ [14.6-26.6\%], $P=0.01 ; 25 \%$ of dalbavancin value, $15.3 \%$ ) (Table 3 , Fig. 3a). No significant reduction in metabolic activity was detected during either of the two periods (months 3 and 6) (Table 3, Fig. 3b).

\section{DISCUSSION}

Enterococci biofilms were reduced in terms of cfu counts and metabolic activity with a 6-month frozen dalbavancin lock solution. However, the addition of heparin may have a 


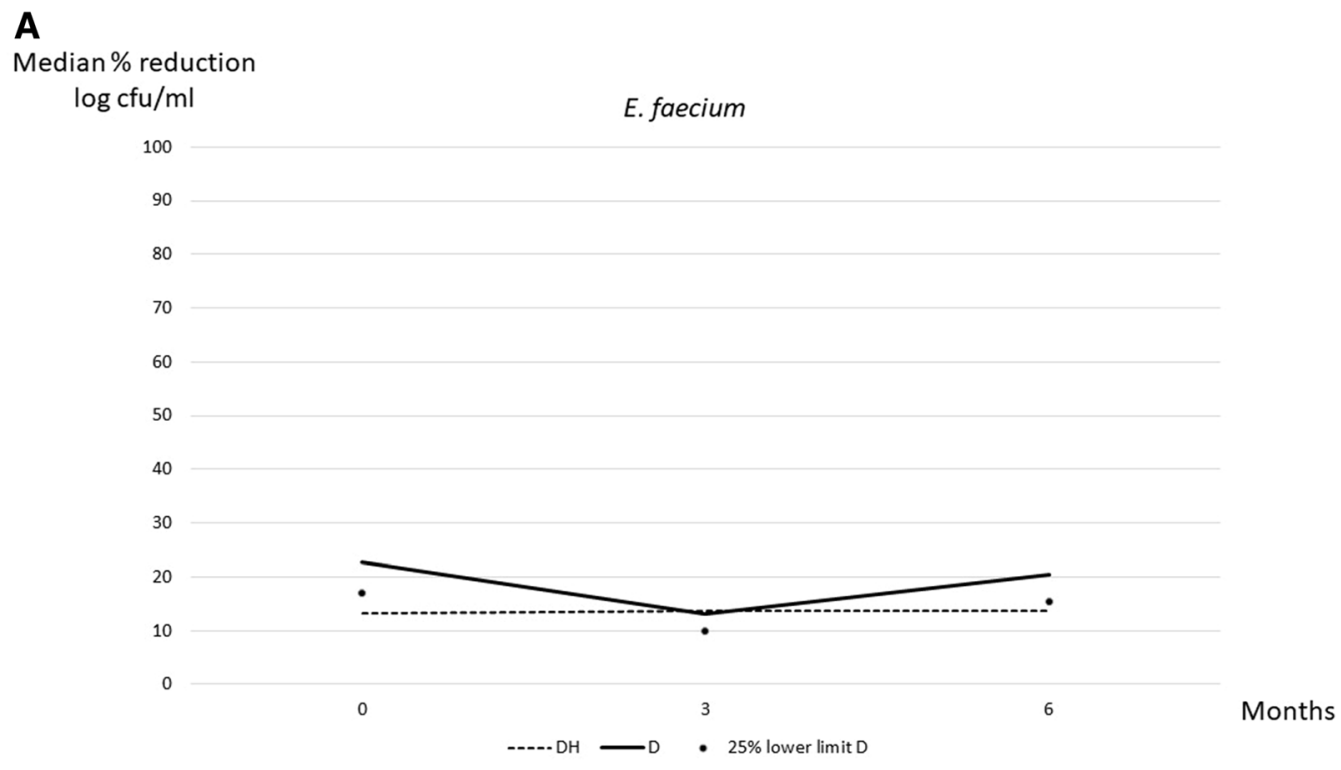

B

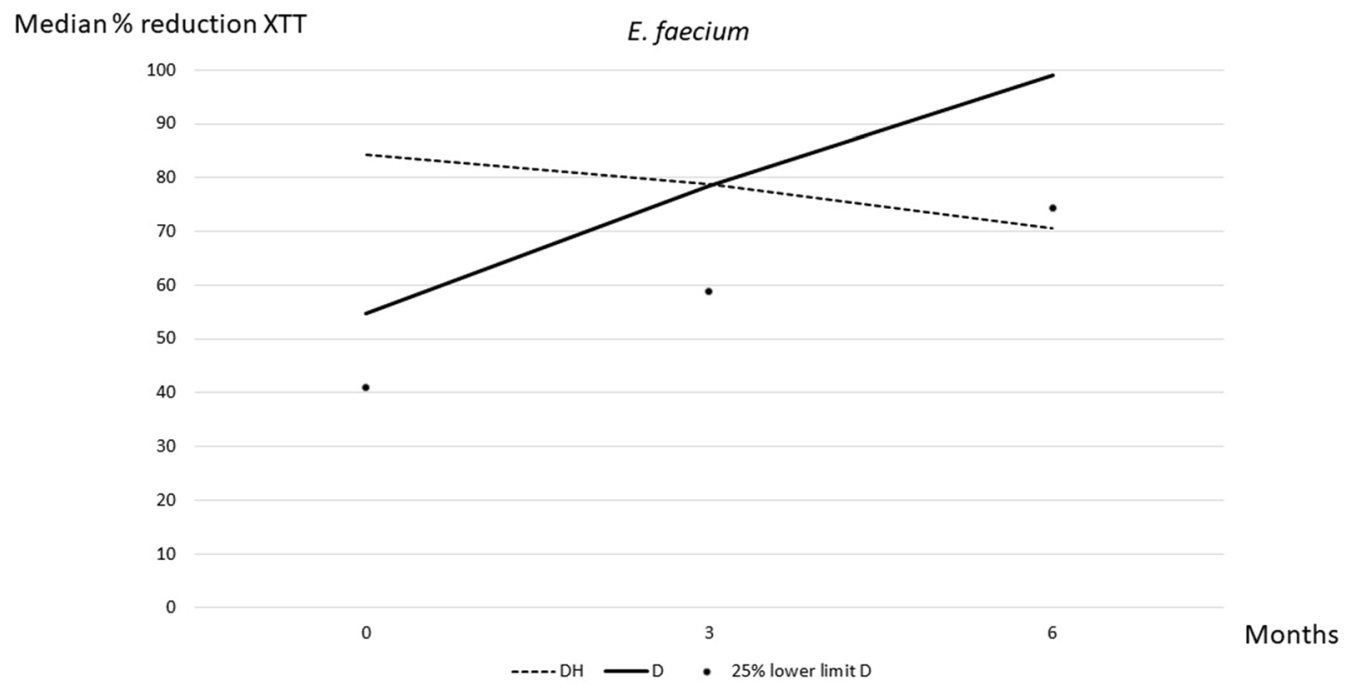

Fig. 2 Median (IQR) percentage reduction in $\log \mathrm{cfu} / \mathrm{ml}$ and metabolic activity for dalbavancin-heparin solution against enterococci according to freezing time. a E. faecalis. b E. faecium. cfu colony-forming units

negative impact on dalbavancin activity during freezing.

C-RBSI can be managed by combining systemic and catheter lock therapies in some circumstances in which the catheter must be retained [4-9]. Current international and Spanish guidelines for the management of catheter-related infections recommend few antimicrobials for the treatment of Gram-positive C-RBSI episodes [14, 15]. Therefore, research with new lipoglycopeptides is needed for catheter salvage attempts, as only one unpublished study has addressed the possible use of dalbavancin as a lock solution [22].

Our group previously demonstrated the antibiofilm activity of a DH catheter lock solution as 
Table 3 Median (IQR) percentage reduction in $\log c f u / m l$ and metabolic activity for dalbavancin alone and dalbavancin-heparin against Enterococcus faecalis and Enterococcus faecium during freezing

\begin{tabular}{llllll}
\hline & Month & D & DH & $P^{*}$ & $\begin{array}{c}\text { 25\% lower } \\
\text { limit of D }\end{array}$ \\
\hline Enterococcus faecalis & & & & & \\
Median (IQR) \% reduction in log cfu/ml & 0 & $22.9(19.8-30.8)$ & $10.7(4.3-19.4)$ & $\mathbf{0 . 0 1}$ & 17.2 \\
& 3 & $22.8(20.1-34.5)$ & $13.0(9.2-19.1)$ & $\mathbf{0 . 0 0 1}$ & 17.1 \\
& 6 & $13.4(12.5-20.2)$ & $9.1(7.9-12.5)$ & $\mathbf{0 . 0 0 1}$ & 10.1 \\
Median (IQR) \% reduction in XTT & 0 & $81.9(56.6-89.8)$ & $77.8(60.6-80.3)$ & 0.45 & 61.4 \\
& 3 & $77.6(67.1-93.1)$ & $80.7(69.3-94.2)$ & 0.76 & 58.2 \\
Enterococcus faecium & 6 & $95.9(90.5-98.7)$ & $74.8(61.3-86.00)$ & 0.002 & 71.9 \\
Median (IQR) \% reduction in log cfu/ml & 0 & $22.7(14.1-24.9)$ & $13.2(11.9-20.6)$ & 0.20 & 17.1 \\
& 3 & $13.1(8.1-30.5)$ & $13.7(0.0-21.9)$ & 0.48 & 9.8 \\
& 6 & $20.4(14.6-26.6)$ & $13.6(13.0-18.9)$ & $\mathbf{0 . 0 1}$ & 15.3 \\
Median (IQR) \% reduction in XTT & 0 & $54.7(39.1-68.7)$ & $84.4(71.8-93.5)$ & 0.002 & 41.0 \\
& 3 & $78.5(30.8-74.2)$ & $78.9(71.3-86.7)$ & 0.27 & 58.9 \\
& 6 & $60.3(15.3-76.00)$ & $70.7(55.3-85.8)$ & 0.16 & 45.2 \\
\hline
\end{tabular}

$S D$ standard deviation, $D$ dalbavancin, $D H$ dalbavancin-heparin, $c f u$ colony-forming units, $I Q R$ interquartile range

*Values in bold represent clinical significance (median \% reduction of DH values were below 25\% lower limit of median \% reduction of $\mathrm{D}$ values)

an alternative to vancomycin against Grampositive bacteria and found that methicillin-resistant Staphylococcus aureus and Staphylococcus epidermidis biofilms were successfully reduced, even when DH solution was frozen for up to 6 months $[17,18]$.

Although several in vitro studies have demonstrated the anti-biofilm activity of dalbavancin against staphylococci, little is known about its efficacy against enterococcal biofilms $[16,23-25]$. Only one study has assessed the in vitro activity of dalbavancin against enterococcal biofilms. The authors found the drug to be active against the 83 tested vancomycinsusceptible isolates both in planktonic and biofilm forms, suggesting that it could be used in a catheter lock solution for enterococcal C-RBSI, in particular only for vancomycin- susceptible enterococci [26]. Therefore, we also included E. faecalis and E. faecium strains in our experiments.

Our results show that the anti-biofilm effect of a frozen dalbavancin solution was not affected in terms of $\log \mathrm{cfu} / \mathrm{ml}$ and metabolic activity in the eight enterococcal strains we tested. When heparin was added to dalbavancin during freezing, a significant loss of efficacy was observed in E. faecalis according to the median percentage reduction in metabolic activity at the end of the experiment (month 6, 51.8\%) compared with baseline (month 0, 84.4\%). Comparison of the anti-biofilm effect of $\mathrm{DH}$ observed in this study and the results of our previous study on staphylococci revealed that the percentage reduction ranges in $\log \mathrm{cfu} / \mathrm{ml}$ (enterococci, 9.1-13.7\%; staphylococci, 


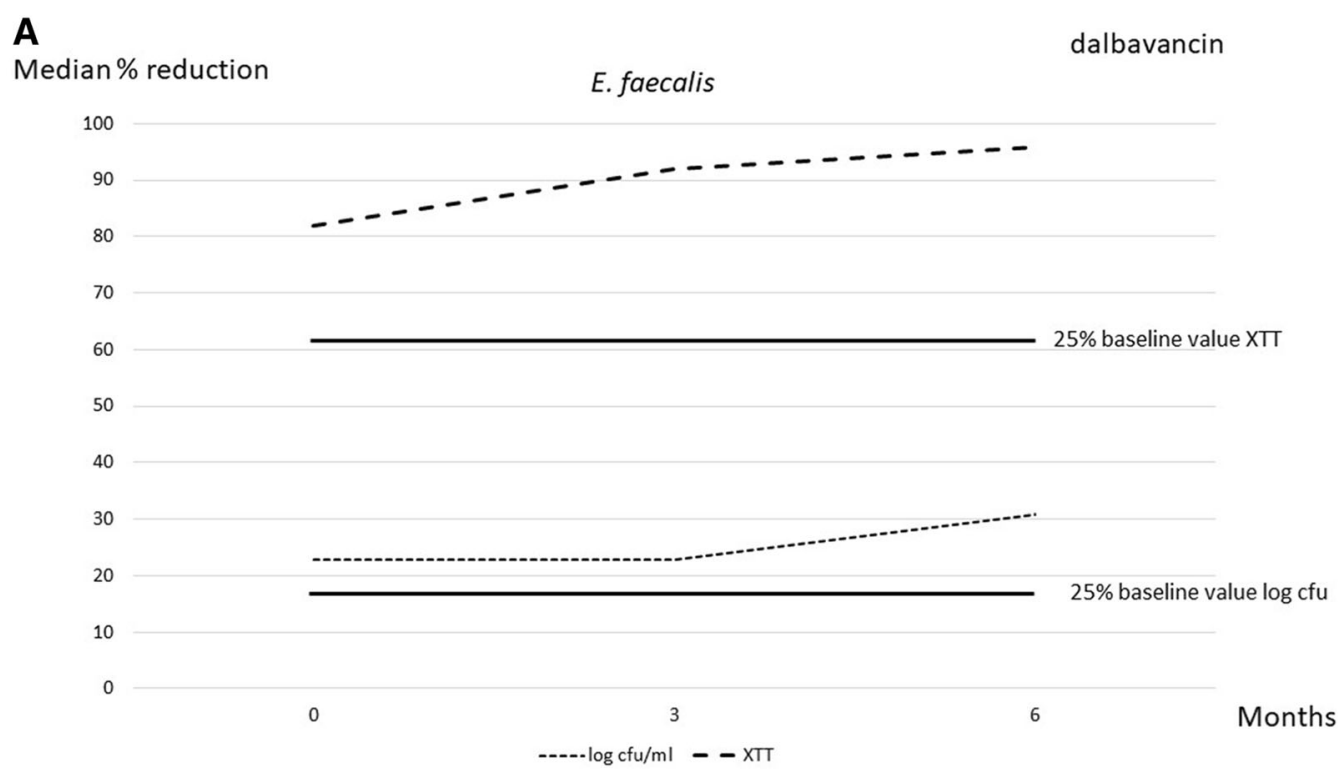

B
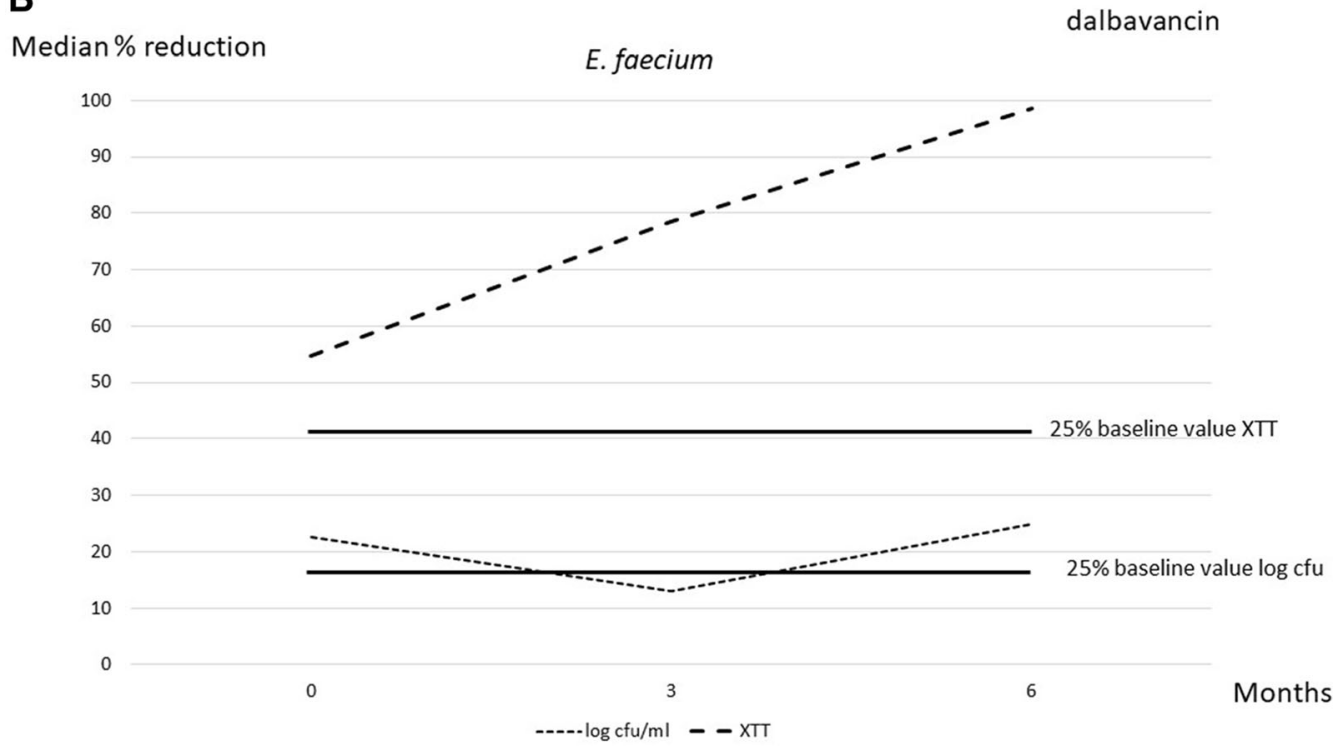

Fig. 3 Median (IQR) \% reduction in a $\log c f u / m l$ and b metabolic activity for dalbavancin and dalbavancin-heparin solutions against $E$. faecalis according to freezing time. Median (IQR) \% reduction in c $\log \mathrm{cfu} / \mathrm{ml}$ and

9.1-29.1\%) and metabolic activity (enterococci, 51.8-84.4\%; staphylococci, 58.9-100\%) were similar between them, even though the statistical analysis was based on a comparison of mean (SD) values using linear mixed models [18]. d metabolic activity for dalbavancin and dalbavancin-heparin solutions against E. faecium according to freezing time. DH dalbavancin-heparin, D dalbavancin, cfu colony-forming units

We assessed the efficacy of dalbavancin in enterococcal biofilms. However, we also analyzed whether heparin affected bioactivity when combined in a catheter lock solution both before and after the freezing period. We found-at least in terms of cfu counts-a 

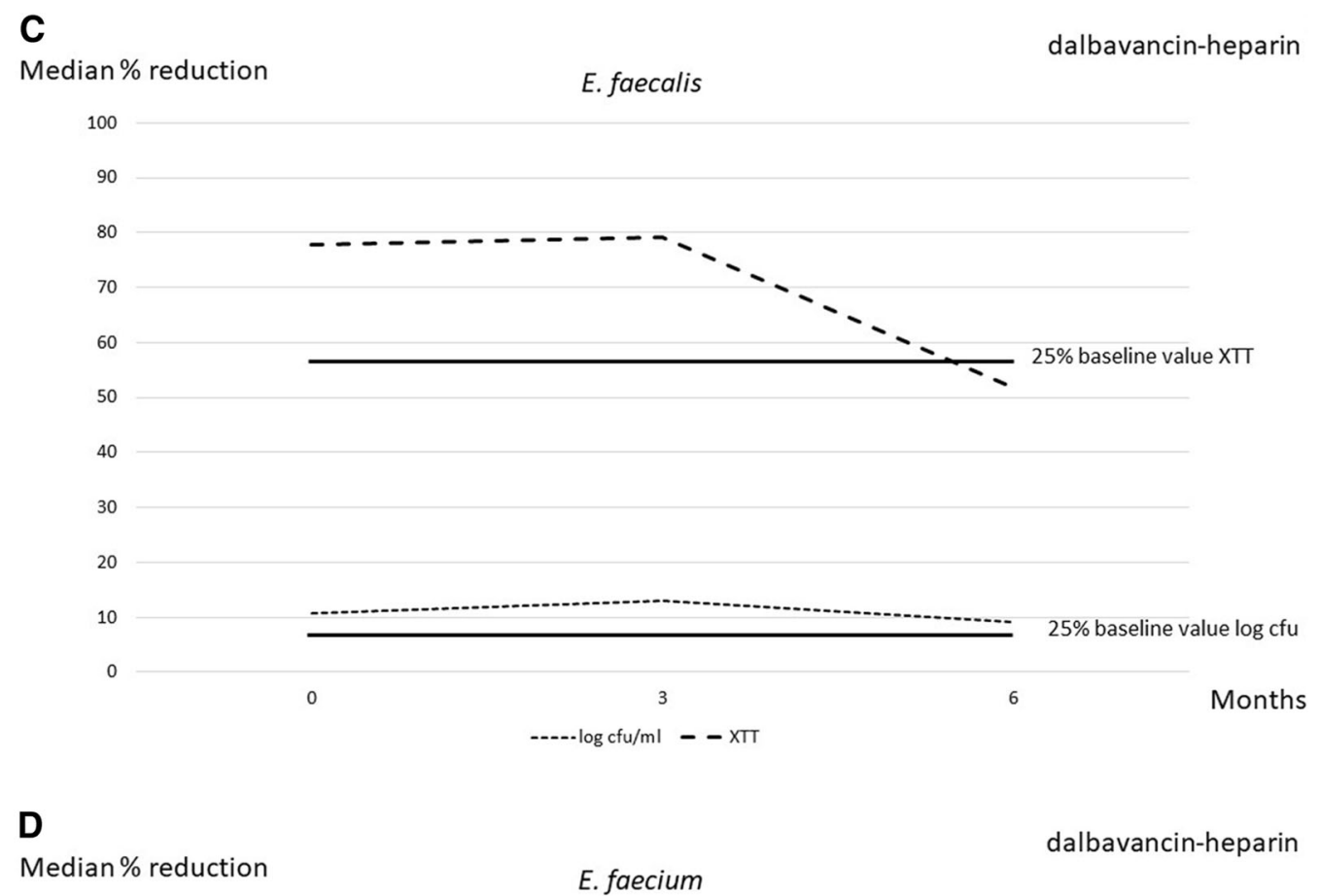

Median \% reduction

E. faecium



Fig. 3 continued

statistically and clinically significant reduction in the bioactivity of dalbavancin when it was frozen with heparin compared with dalbavancin alone (E. faecalis, $9.1 \%$ vs. $13.4 \%$, $P=0.001 ; \quad E$. faecium, $13.6 \%$ vs. $20.4 \%$, $P=0.01)$. Therefore, in our opinion, a catheter lock attempt with dalbavancin against C-RBSI caused by enterococci may be performed using a frozen dalbavancin solution without heparin. While it would be more useful and easier to use a frozen vial containing both dalbavancin and heparin, in these circumstances in which we demonstrated that heparin negatively affects dalbavancin activity under freezing, 60 IU of heparin should be added to the frozen dalbavancin solution at the time of catheter lock.

One of the main limitations of the study is that we arbitrarily define "clinical significance" on the basis of a previous study in which a reduction of dalbavancin bioactivity greater 
than $25 \%$ was reasonable enough to consider it a relevant cutoff above which it may have "clinical impact". Moreover, despite the inclusion of high biofilm producer-clinical strains isolated from blood of patients with C-RBSI, conclusions must be carefully interpreted, as further studies are needed to validate it with large number of isolates.

\section{CONCLUSION}

We confirm that the bioactivity of a dalbavancin catheter lock solution against enterococcal biofilms was not reduced after 6 months of freezing. However, the addition of heparin could negatively affect it. We consider that heparin should be added to frozen dalbavancin at the time of catheter lock.

\section{ACKNOWLEDGEMENTS}

We thank Thomas O'Boyle for his help in the preparation of the manuscript.

Funding. M. Guembe is supported by the Miguel Servet Program (ISCIII-MICINN, MS13/ 00268) of the Health Research Fund (FIS) of the Carlos III Health Institute (ISCIII), Madrid, Spain. M. Díaz-Navarro is supported by the Consejería de Educación, Juventud y Deporte de la Comunidad de Madrid and Fondo Social Europeo (PEJD-2020-AI_BMD-17971). The study was partially financed by grants from the ISCIII (PI18/00045) and the European Regional Development Fund (FEDER) "A way of making Europe". The Journal's Rapid Service Fee was funded by the authors.

Authorship. All named authors meet the International Committee of Medical Journal Editors (ICMJE) criteria for authorship for this article, take responsibility for the integrity of the work as a whole, and have given their approval for this version to be published.
Author Contributions. María Guembe, Marta Díaz-Navarro and María J. Pérez-Granda were responsible for the design and coordination of the study. María Guembe was the chief investigator and responsible for the data analysis. Rama Hafian, Irene Manzano, and Marta Díaz-Navarro developed the experimental model and data collection. María J. PérezGranda, Emilia Cercenado, Carmen Rodríguez and Patricia Muñoz collaborated in the interpretation of the data. All authors contributed to the writing of the final manuscript.

Disclosures. Marta Díaz-Navarro, Rama Hafian, Irene Manzano, María J. Pérez-Granda, Emilia Cercenado, Cristina Pascual, Carmen Rodríguez, Patricia Muñoz, and María Guembe have nothing to disclose.

Compliance with Ethics Guidelines. The study was considered to be exempt for ethical approval by our local ethics committee since it was an in vitro retrospective study.

Data Availability. The datasets generated during and/or analyzed during the current study are available from the corresponding author on reasonable request.

Open Access. This article is licensed under a Creative Commons Attribution-NonCommercial 4.0 International License, which permits any non-commercial use, sharing, adaptation, distribution and reproduction in any medium or format, as long as you give appropriate credit to the original author(s) and the source, provide a link to the Creative Commons licence, and indicate if changes were made. The images or other third party material in this article are included in the article's Creative Commons licence, unless indicated otherwise in a credit line to the material. If material is not included in the article's Creative Commons licence and your intended use is not permitted by statutory regulation or exceeds the permitted use, you will need to obtain permission directly from the copyright holder. To view a copy of this licence, visit http://creativecommons.org/licenses/bync/4.0/. 


\section{REFERENCES}

1. Zhong $\mathrm{Y}$, Zhou L, Liu X, et al. Incidence, risk factors, and attributable mortality of catheter-related bloodstream infections in the intensive care unit after suspected catheters infection: a retrospective 10-year cohort study. Infect Dis Ther. 2021;10(2): 985-99.

2. Cantón-Bulnes ML, Garnacho-Montero J. Practical approach to the management of catheter-related bloodstream infection. Rev Esp Quimioter. 2019;32 Suppl 2(Suppl 2):38-41.

3. Tatarelli P, Parisini A, Del Bono V, Mikulska M, Viscoli C. Efficacy of daptomycin lock therapy in the treatment of bloodstream infections related to long-term catheter. Infection. 2015;43(1):107-9.

4. Beigi AA, Khansoltani S, Masoudpour H, Atapour AA, Eshaghian A, Khademi EF. Influence of intralumenal and antibiotic-lock of vancomycin on the rate of catheter removal in the patients with permanent hemodialysis catheters. Saudi J Kidney Dis Transpl. 2010;21(1):54-8.

5. Krishnasami Z, Carlton D, Bimbo L, et al. Management of hemodialysis catheter-related bacteremia with an adjunctive antibiotic lock solution. Kidney Int. 2002;61(3):1136-42.

6. Del Pozo JL, Alonso M, Serrera A, Hernaez S, Aguinaga A, Leiva J. Effectiveness of the antibiotic lock therapy for the treatment of port-related enterococci, Gram-negative, or Gram-positive bacilli bloodstream infections. Diagn Microbiol Infect Dis. 2009;63(2):208-12.

7. Fortún J, Grill F, Martín-Dávila P, et al. Treatment of long-term intravascular catheter-related bacteraemia with antibiotic-lock therapy. J Antimicrob Chemother. 2006;58(4):816-21.

8. Park $\mathrm{KH}$, Cho $\mathrm{OH}$, Lee SO, et al. Outcome of attempted Hickman catheter salvage in febrile neutropenic cancer patients with Staphylococcus aureus bacteremia. Ann Hematol. 2010;89(11): 1163-9.

9. Kim SH, Kang CI, Kim HB, et al. Outcomes of Hickman catheter salvage in febrile neutropenic cancer patients with Staphylococcus aureus bacteremia. Infect Control Hosp Epidemiol. 2003;24(12):897-904.

10. Foresti S, Di Bella S, Rovelli A, et al. Tigecycline lock therapy for catheter-related bloodstream infection caused by KPC-Producing Klebsiella pneumoniae in two pediatric hematological patients. Antimicrob Agents Chemother. 2015;59(12):7919-20.
11. Funalleras G, Fernández-Hidalgo N, Borrego A, et al. Effectiveness of antibiotic-lock therapy for longterm catheter-related bacteremia due to Gram-negative bacilli: a prospective observational study. Clin Infect Dis. 2011;53(9):e129-32.

12. Peterson WJ, Maya ID, Carlton D, Estrada E, Allon M. Treatment of dialysis catheter-related Enterococcus bacteremia with an antibiotic lock: a quality improvement report. Am J Kidney Dis. 2009;53(1): 107-11.

13. Yen HW, Yang WC, Tarng DC, et al. Daptomycin antibiotic lock therapy for hemodialysis patients with Gram-positive bloodstream infections following use of tunneled, cuffed hemodialysis catheters: retrospective single center analysis. Hemodial Int. 2016;20(2):315-20.

14. Mermel LA, Allon M, Bouza E, et al. Clinical practice guidelines for the diagnosis and management of intravascular catheter-related infection: 2009 update by the Infectious Diseases Society of America. Clin Infect Dis. 2009;49(1):1-45.

15. Chaves F, Garnacho-Montero J, Del Pozo JL, et al. Executive summary: diagnosis and treatment of catheter-related bloodstream infection: clinical guidelines of the Spanish Society of Clinical Microbiology and Infectious Diseases (SEIMC) and the Spanish Society of Intensive Care Medicine and Coronary Units (SEMICYUC). Enferm Infecciosas Microbiol Clin (Eng Ed). 2018;36(2):112-9.

16. Meeker DG, Beenken KE, Mills WB, et al. Evaluation of antibiotics active against methicillin-resistant Staphylococcus aureus based on activity in an established biofilm. Antimicrob Agents Chemother. 2016;60(10):5688-94.

17. Díaz-Ruíz C, Alonso B, Cercenado E, et al. Can dalbavancin be used as a catheter lock solution? J Med Microbiol. 2018;67(7):936-44.

18. Rubia M, Cordero A, Pérez-Granda MJ, Cercenado E, et al. In vitro study to evaluate the bioactivity of freezing a heparin-based dalbavancin lock solution. Antimicrob Agents Chemother. 2020;64(12): e01495-20.

19. Alonso B, Pérez-Granda MJ, Latorre MC, et al. Is heparinized $40 \%$ ethanol lock solution efficient for reducing bacterial and fungal biofilms in an in vitro model? PLoS ONE. 2019;14(7):e0219098.

20. Clinical and Laboratory Standards Institute. Methods for dilution antimicrobial susceptibility tests for bacteria that grow aerobically; approved standard. 10th ed. CLSI document M07-A10. Wayne: Clinical and Laboratory Standards Institute; 2017. 
21. Peeters E, Nelis HJ, Coenye T. Comparison of multiple methods for quantification of microbial biofilms grown in microtiter plates. J Microbiol Methods. 2008;72(2):157-65.

22. Sevillano DAL, González N, Buenache N, et al. Evaluación comparativa de la actividad de la dalbavancina, daptomicina, linezolid y vancomicina en la estrategia de sellado del catéter colonizado por S. aureus resistente a meticilina (SARM) y de S. epidermidis resistente a meticilina (SEMR). Congreso de la Sociedad Española de Enfermedades Infecciosas y Microbiología Clínica (SEIMC). 2017.

23. Baldoni D, Furustrand Tafin U, et al. Activity of dalbavancin, alone and in combination with rifampicin, against meticillin-resistant Staphylococcus aureus in a foreign-body infection model. Int J Antimicrob Agents. 2013;42(3):220-5.

24. Fernández J, Greenwood-Quaintance KE, Patel R. In vitro activity of dalbavancin against biofilms of staphylococci isolated from prosthetic joint infections. Diagn Microbiol Infect Dis. 2016;85(4): 449-51.

25. Knafl D, Tobudic S, Cheng SC, Bellamy DR, Thalhammer F. Dalbavancin reduces biofilms of methicillin-resistant Staphylococcus aureus (MRSA) and methicillin-resistant Staphylococcus epidermidis (MRSE). Eur J Clin Microbiol Infect Dis. 2017;36(4): 677-80.

26. Neudorfer K, Schmidt-Malan SM, Patel R. Dalbavancin is active in vitro against biofilms formed by dalbavancin-susceptible enterococci. Diagn Microbiol Infect Dis. 2018;90(1):58-63.

\section{Publisher's Note}

Springer Nature remains neutral with regard to jurisdictional claims in published maps and institutional affiliations. 